\title{
Transcriptomic changes in the root of oil palm (Elaeis guineensis Jacq.) upon inoculation with Bacillus sphaericus UPMB10
}

\begin{abstract}
Plant growth-promoting bacteria (PGPB) promote plant growth through a combination of mechanisms which affect mineral nutrition and root development. In this study, we profiled the transcripts in oil palm roots inoculated with a PGPB, Bacillus sphaericus UPMB10, upon the establishment of a stable inoculum on the root surface by cDNA microarray analysis. A total of 70 from more than 3,700 transcripts examined were found to be significantly upregulated in the roots of oil palm inoculated with B. sphaericus UPMB10 compared to those from uninoculated oil palm roots. Majority of these genes were related to protein synthesis and processing, stress, primary metabolism, and membrane transport. Many ribosomal proteins were upregulated in the roots of B. sphaericusinoculated oil palm plantlets. In addition, B. sphaericus UPMB10 were able to upregulate the expression of a gene encoding low affinity nitrate transporter, independent of root morphogenesis. These rhizosphere bacteria were also demonstrated to induce stress-related proteins that may lead to tolerance to biotic and abiotic stresses.
\end{abstract}

Keyword: Bacillus sphaericus; cDNA microarray; Nitr 